TUM-HEP-249/96

hep-ph/9608356

\title{
Higher-order corrections in the SM Higgs sector: the right scale *
}

\author{
Kurt Riesselmann ${ }^{\text {a }}$ \\ aPhysik Department, Technische Universität München, \\ James-Franck-Str., D-85748 Garching, Germany
}

The evaluation of high-energy cross sections involving the SM Higgs boson requires the use of the Higgs running coupling $\lambda(\mu)$. Taking $\mu$ to be equal to the center-of-mass energy $\sqrt{s}$ of the scattering process, the perturbative approach fails for relatively small values of the Higgs mass and coupling, $\lambda(\sqrt{s}) \approx 2.2$. Performing an approximate resummation of "bubble" Feynman diagrams, we find the scale $\mu=\sqrt{s} / \mathrm{e} \approx \sqrt{s} / 2.7$ to yield reliable perturbative results, even for large Higgs mass and coupling. The new perturbative upper limit on the Higgs running coupling is $\lambda(\sqrt{s} / \mathrm{e}) \approx 4$.

\section{Physical motivation}

The Higgs boson of the Standard Model (SM) still eludes detection. Once a Higgs boson has been discovered, its couplings to other particles as well as its self-couplings need to be measured and compared with SM predictions. Of particular interest are the couplings of the Higgs boson to the (longitudinally polarized) gauge bosons $W^{+}, W^{-}, Z$ of the SM. Scattering processes involving these particles receive contributions from the gauge couplings (which already can be tested at LEP2) as well as the trilinear and quartic Higgs couplings. To test the latter, reliable predictions for SM cross sections and decay widths are important. In the case of high-energy scattering processes, the resummation procedure described here is essential. The prospects for studying the various couplings at future colliders are, for example, discussed in 1 . 5 .

At the same time, physical observables involving Higgs couplings can be used to derive upper bounds on a perturbative Higgs coupling. The size of the radiative corrections, the sensitivity of the result to the renormalization scale $\mu$, and the perturbative violation of unitarity give upper limits on the values of the Higgs mass $M_{H}$ and the

\footnotetext{
*To appear in the Proceedings of the Workshop "QCD and QED in Higher Order", Rheinsberg, Germany (April 1996).
}

Higgs quartic coupling $\lambda$ beyond which perturbation theory fails. Using high-energy amplitudes, rather stringent bounds on the Higgs quartic coupling have been derived [6 9]. We show that these bounds are relaxed when the right choice of scale is chosen in connection with the running coupling.

\section{The Higgs running coupling}

In the SM, the value of the quartic Higgs coupling $\lambda$ is fixed at tree level by the value of the Higgs mass: $\lambda=M_{H}^{2} / 2 v^{2}$, where $v=246 \mathrm{GeV}$ is the vacuum expectation value. Calculating physical observables with energy scales larger than $M_{H}$, renormalization group methods suggest the use of the running coupling $\lambda(\mu)$. Fixing the running coupling at the scale $\mu=M_{H}$ such that it equals the tree level result,

$\lambda\left(M_{H}\right)=\frac{M_{H}^{2}}{2 v^{2}}$,

the value of the running coupling at scales $\mu>$ $M_{H}$ is fixed by the $\beta$ function of the theory. Neglecting gauge and Yukawa couplings, a good approximation for a heavy Higgs boson, the oneloop result for the running coupling is

$$
\lambda(\mu)=\frac{\lambda\left(M_{H}\right)}{1-\frac{\beta_{0}}{2} \frac{\lambda\left(M_{H}\right)}{16 \pi^{2}} \ln \left(\frac{\mu^{2}}{M_{H}^{2}}\right)}, \quad \beta_{0}=24 .
$$


Solutions up to three loops are discussed in [9]. From the previous equations it is apparent that perturbation theory will cease to be useful if either the Higgs mass $M_{H}$ is large or if the energy scale $\mu$ of a process is large.

\section{Two-loop results in the Higgs sector}

Several observables related to the SM Higgs sector have been calculated to two loops in perturbation theory using the heavy-Higgs limit. Neglecting the subleading corrections due to gauge and Yukawa couplings, the two-loop $O\left(\lambda^{2}\right)$ results for the partial widths of the Higgs boson decaying into a pair of gauge bosons [10,11] or a pair of fermions 12,13 are:

$$
\begin{aligned}
& \Gamma\left(H \rightarrow W^{+} W^{-}\right) \propto \\
& \lambda\left(M_{H}\right)\left(1+2.8 \frac{\lambda\left(M_{H}\right)}{16 \pi^{2}}+62.1 \frac{\lambda^{2}\left(M_{H}\right)}{\left(16 \pi^{2}\right)^{2}}\right), \\
& \Gamma(H \rightarrow f \bar{f}) \propto \\
& \quad g_{f}^{2}\left(1+2.1 \frac{\lambda\left(M_{H}\right)}{16 \pi^{2}}-32.7 \frac{\lambda^{2}\left(M_{H}\right)}{\left(16 \pi^{2}\right)^{2}}\right) .
\end{aligned}
$$

Here $g_{f}$ is the Yukawa coupling of the fermion $f$. Comparing the magnitude of the one-loop and two-loop corrections, perturbation theory seems to work up to values of $\lambda\left(M_{H}\right) \approx 7$, that is, Higgs masses of about $1 \mathrm{TeV}$. However, an analysis of the scale- and scheme-dependence [9] reveals that higher-order terms may spoil perturbation theory already for values of $\lambda\left(M_{H}\right) \approx 4$, or equivalently, $M_{H} \approx 700 \mathrm{GeV}$.

Perturbation theory fails even sooner in the case of high-energy scattering processes. For example, the two-loop correction to the scattering process $W_{L}^{+} W_{L}^{-} \rightarrow Z_{L} Z_{L}$ is 8, 14,

$$
\begin{aligned}
\sigma\left(W_{L}^{+} W_{L}^{-} \rightarrow Z_{L} Z_{L}\right) \propto \\
\lambda^{2}(\sqrt{s})\left(1-42.6 \frac{\lambda(\sqrt{s})}{16 \pi^{2}}+2457.9 \frac{\lambda^{2}(\sqrt{s})}{\left(16 \pi^{2}\right)^{2}}\right)(5)
\end{aligned}
$$

where the subscript $L$ denotes the longitudinal polarization of the gauge bosons, and $\sqrt{s}$ is the center-of-mass energy of the incoming particles. The assumption $\sqrt{s} \gg M_{H}$ used in calculating (5) is valid if $\sqrt{s}>2-3 M_{H}$ [8]. It is striking that the coefficients appearing in the cross section are much larger than the corresponding coefficients of the decay widths. Consequently, the upper bound on a perturbative Higgs coupling is stronger than in the case of the decay widths. Comparing the size of the one-loop and two-loop cross section, a bound of $\lambda(\sqrt{s}) \approx 2.2$ is found [8]. A similar bound is also derived when investigating the scheme- and scale-dependence of the cross section [9]. Since $\lambda(\sqrt{s})>\lambda\left(M_{H}\right)$ for $\sqrt{s}>M_{H}$, the bound $\lambda(\sqrt{s}) \approx 2.2$ represents a strong restriction on the Higgs mass. Choosing $\sqrt{s}$ to be a couple of $\mathrm{TeV}$, the Higgs mass has to be less than about $400 \mathrm{GeV}$ to allow for a perturbative calculation of the cross section.

The cross sections for low energies $\left(\sqrt{s}<M_{H}\right)$ and energies near the resonance $\left(\sqrt{s} \approx M_{H}\right)$ have been calculated including one-loop corrections [15 17]. The calculation requires the inclusion of trilinear Higgs coupling. The one-loop results do not yield stringent bounds on the Higgs coupling. A two-loop calculation is not yet available.

\section{Resummation of bubble contributions: the right scale for scattering processes}

We are able to improve the perturbative character of the cross section (5) significantly by introducing a resummation of nonlogarithmic terms.

\subsection{Identification of bubble contributions}

The large size of the one- and two-loop coefficients in (5) can be traced back to the finite pieces of the scattering graphs which contribute to the amplitude. In the limit $\sqrt{s} \gg M_{H}$, all oneloop scattering graphs can be written in terms of the massless bubble Feynman diagram $B\left(p^{2}\right)$, the one-loop two-point function with two massless propagators. The quantity $p^{2}$ is the fourmomentum squared running through the bubble. Using the usual Mandelstam variables $s, t, u$, the high-energy amplitude of the scattering process considered above can be written as

$$
\begin{aligned}
& a\left(W_{L}^{+} W_{L}^{-} \rightarrow Z_{L} Z_{L}\right)= \\
& \quad-\lambda(\mu)\left[2+O\left(M_{H}^{2} / s\right)\right] \\
& \quad-\lambda^{2}(\mu)\left[16 B(s)+4 B(t)+4 B(u)+O\left(M_{H}^{2} / s\right)\right] \\
& \quad+\text { counterterms }+ \text { wavefct.renorm. . }
\end{aligned}
$$

The counterterms and wavefunction renormalizations are independent of $s, t, u$. The massless oneloop bubble is evaluated in dimensional regular- 
ization. Choosing the dimension to be $D=4-2 \epsilon$, the expression for $B\left(p^{2}\right)$ equals

$B\left(p^{2}\right)=$

$\frac{\left(4 \pi e^{-\gamma}\right)^{\epsilon}}{16 \pi^{2}}\left(\frac{1}{\epsilon}+\ln \frac{\mu^{2}}{\left|p^{2}\right|}+2+i \pi \Theta\left(p^{2}\right)+O(\epsilon)\right)$.

The step function $\Theta$ ensures that only the $s$ channel gives rise to an imaginary part.

The variables $t$ and $u$ are proportional to $s$ and contribute to the logarithmic $s$-dependence of the amplitude due to the bubble diagrams. The coeffcients of all $s$-dependent logarithms add up to the one-loop beta function coefficient $\beta_{0}=24$. The complete resummation of these leading logarithms $\ln (s)$ is achieved by setting the scale $\mu$ of the running coupling to be equal to $\sqrt{s}$, the standard choice in renormalization group procedures. Nonlogarithmic pieces do not get resummed when making this choice.

Looking at (耳) we find that the logarithm is accompanied by a constant 2 . While the imaginary part of the bubble only contributes to the $s$-channel, the constant 2 is universal to $s, t$, and $u$ channel. It contributes to the one-loop amplitude a term $2 \beta_{0}=48$. Including counterterms and wavefunction renormalizations, the complete high-energy amplitude is

$$
\begin{aligned}
a=-2 \lambda(\mu)+\frac{\lambda^{2}(\mu)}{16 \pi^{2}} & {\left[\beta_{0}\left(\ln \frac{\mu^{2}}{s}+2\right)+16 i \pi\right.} \\
& \left.-4 \ln \frac{\sin ^{2} \theta}{4}-13.35\right]
\end{aligned}
$$

where $\theta$ denotes the c.m.s. scattering angle which relates $t$ and $u$ to $s$. We find that the contribution

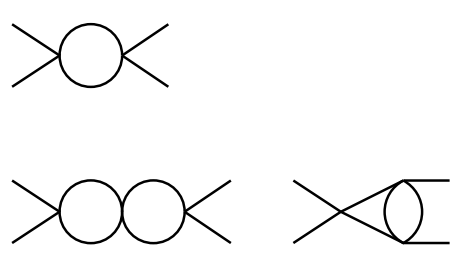

Figure 1. Topologies of $s$-channel Feynman diagrams contributing to high-energy $W_{L}^{+} W_{L}^{-} \rightarrow$ $Z_{L} Z_{L}$ scattering at one loop (top row) and two loops (bottom row). from the 2's dominates the total one-loop correction of the amplitude and the cross section. It is important to note that each logarithm of the oneloop amplitude is accompanied by the constant 2 and that there is no further contribution proportional to $\beta_{0}$. In particular, the counterterms and wavefunction renormalizations in (6) are evaluated from a variety of different low-energy Feynman diagrams, all of which have different nonlogarithmic pieces. Details are given in [18].

At higher orders in perturbation theory, the "massless bubble structure" of the high-energy scattering graphs persists. This is due to the $\Phi^{4}$-theory nature of the high-energy interactions in the SM Higgs sector. In Fig. 11 we see the $s$ channel Feynman diagrams of the $2 \rightarrow 2$ amplitude at one and two loops. The $t$ - and $u$-channel contributions are not shown. They are obtained using crossing symmetries of the external legs. At one loop, the bubble is the only scattering topology, yielding the contributions according to (6). At two loops, we encounter two topologies: the squared bubble topology $B^{2}\left(p^{2}\right)$ and the insertion of a bubble at the vertex of a second bubble. In the case of the topology $B^{2}$, each bubble gives rise to a constant 2 . In the second topology, only the inner bubble features a constant 2. The integration of the outer loop is not a simple bubble integral. It yields a finite piece different from 2 .

The situation is similar at three loops and beyond. At $n$ loops there always is a topology which is a product of $n$ bubbles. Next there are topologies which have $n-1$ bubbles, with the final integration being a modified bubble integral. Then there are topologies with $n-2$ bubbles, and so on. Starting at three loops, there also exist nonplanar graphs which cannot be naturally viewed as being constructed from bubble graphs. Yet their weight is expected to be small compared to the numerous bubble related contributions to the complete set of $n$-loop diagrams.

\subsection{The right scale}

The above arguments suggest that the constant 2 accompanies the majority of the leading logarithms. The resummation of this contribution is desirable. Since the $s$-dependend leading logarithms are resummed using the choice $\mu=\sqrt{s}$, it is easy to adjust $\mu$ to also yield a resummation of 
the "leading $2 "$ such that

$$
\ln \left(\frac{\mu^{2}}{s}\right)+2=0 .
$$

The right scale choice is thus

$\mu=\frac{\sqrt{s}}{\mathrm{e}} \approx \frac{\sqrt{s}}{2.7}$.

The resulting leading-log (LL) result for the highenergy amplitude is therefore

$a\left(W_{L}^{+} W_{L}^{-} \rightarrow Z_{L} Z_{L}\right)=-2 \lambda(\sqrt{s} / \mathrm{e})$.

\subsection{Testing the new choice of scale}

Such a particular choice of $\mu$ is, of course, only meaningful if one expects it to work at higher orders as well. An exact resummation of subleading logarithms together with nonlogarithmic terms is not possible. All known scale-setting schemes resort to various methods to justify their choice of scale at subleading level; see 19 for a discussion. A first check for the validity of the leading-log scale choice at higher orders is the size of the coefficients at higher orders. A bad choice of scale would reduce the perturbative coefficients in lower orders and push large corrections into higher orders. Taking our scale choice $\mu=\sqrt{s} / \mathrm{e}$, we re-evaluate the next-to-next-toleading-log (NNLL) cross section (5):

$$
\begin{aligned}
& \sigma\left(W_{L}^{+} W_{L}^{-} \rightarrow Z_{L} Z_{L}\right) \propto \\
& \lambda^{2}(\sqrt{s} / \mathrm{e})\left(1+5.4 \frac{\lambda(\sqrt{s} / \mathrm{e})}{16 \pi^{2}}+539.9 \frac{\lambda^{2}(\sqrt{s} / \mathrm{e})}{\left(16 \pi^{2}\right)^{2}}\right) .
\end{aligned}
$$

Comparing the coefficients with the result for $\mu=$ $\sqrt{s}$ in (5) we find that both one-loop and two-loop coefficient are greatly reduced in magnitude. This suggests that our improved scale choice also works at the subleading level.

Another good check on the quality of our scale choice is the variation of the scale around the central value chosen. The explicit $\mu$-dependence of the cross section $\sigma\left(W_{L}^{+} W_{L}^{-} \rightarrow Z_{L} Z_{L}\right)$ can be found in [9]. In Fig. 2 we show the LL, NLL, and NNLL results for $s \sigma$ as a function of $\mu / \sqrt{s}$. The Higgs running coupling is fixed to be $\lambda(\mu=\sqrt{s} / \mathrm{e})=1.5$. ( The coupling at $\mu=\sqrt{s}$ is then fixed by Eq. (2) and yields $\lambda(\sqrt{s})=1.9$.) Such a value corresponds, for example, to a Higgs mass of $390 \mathrm{GeV}$ and $\sqrt{s}=1 \mathrm{TeV}$. Fixing the running coupling at a certain scale, the quantity $s \sigma$

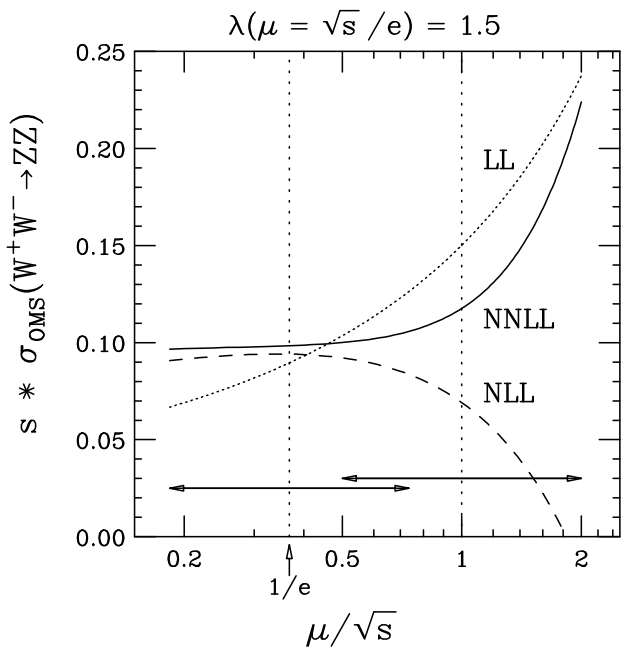

Figure 2. The scaled high-energy cross section of $W_{L}^{+} W_{L}^{-} \rightarrow Z_{L} Z_{L}$ for $\lambda=1.5$. Gauge and Yukawa coupling contributions are neglected. The horizontal lines with arrows indicate the typical variation of $\mu$, taking the central values to be $\mu=\sqrt{s} /$ e and $\mu=\sqrt{s}$.

is a function of the single variable $\mu / \sqrt{s}$. Varying $\mu$ around the central values $\sqrt{s}$ and $\sqrt{s} / \mathrm{e}$ as indicated in Fig. 2, we determine the sensitivity of the result with respect to the renormalization group logarithms. If perturbation theory is to be reliable, one expects an order-by-order reduction of the scale dependence. Comparing the results of our scale choice $\mu=\sqrt{s} / \mathrm{e}$ with the standard choice $\mu=\sqrt{s}$ we find a greatly reduced scale dependence when choosing $\sqrt{s} / \mathrm{e}$. The scale dependence around $\mu=\sqrt{s}$ /e nicely reduces when going from LL to NLL order. At the same time, the magnitude of the one-loop and two-loop corrections is significantly reduced when using our improved scale choice, a fact that we already noticed by comparing (5) and (12).

Looking at Fig. 2 we also see that the NLL cross section can become negative, that is, the magnitude of the one-loop correction exceeds unity if the scale $\mu$ is chosen too large: The perturbative calculation completely fails. The choice $\mu=\sqrt{s} / \mathrm{e}$, however, is clearly in a perturbatively 
reliable region.

\subsection{Upper bound on the running coupling}

Of course, the perturbative behaviour of the cross section also depends on the value of the running coupling. In Fig. 2 we chose $\lambda(\sqrt{s} / \mathrm{e})=1.5$. Increasing this value, perturbation theory will eventually also cease to be useful even when taking our improved choice of scale. We have found that this happens for $\lambda(\sqrt{s} / \mathrm{e}) \approx 4$. Such a value corresponds to, for example, $M_{H}=700 \mathrm{GeV}$ and $\sqrt{s}=1.9 \mathrm{TeV}$. (A larger value of $M_{H}$ results in a smaller value of $\sqrt{s}$, eventually violating the high-energy assumption of the calculation.) The details on obtaining the upper bound are given in 18, where a re-analysis of unitarity violation is carried out. Comparing the new bound with the previous result of $\lambda(\sqrt{s}) \approx 2.2$ [6 9], the perturbative region is significantly extended.

There also exist upper bounds on the Higgs mass and coupling which are derived from the renormalization group equations for the Higgs running coupling. Requiring that the one-loop running coupling $\lambda(\Lambda)$ remains finite up to large embedding scales $\Lambda$, an upper bound on $\lambda\left(M_{H}\right)$ is deduced 20]. These bounds are less stringent than the bounds derived from physical observables such as cross sections and decay widths.

The constraints obtained from lattice calculations are described in [21]. A comparison of perturbative and lattice results is given in [18.

\section{The right scale in Higgs decays}

The necessity of modifying the standard scale choice in scattering processes leads to the question of whether the standard scale choice in decay processes needs to be modified as well. In contrast to scattering processes, the Higgs decays feature a fixed center-of-mass energy of $\sqrt{s}=M_{H}$. The Higgs mass appearing in the Feynman integrals cannot be neglected. Consequently, the different bubble topologies contribute various finite pieces to the one-loop result: no universal term proportional to $\beta_{0}$ arises. This suggests to leave the standard choice $\mu=M_{H}$ unchanged.

Similar to the discussion of the cross section, we can check the correctness of the choice $\mu=M_{H}$ by plotting the $\mu$-dependence of the decay widths.

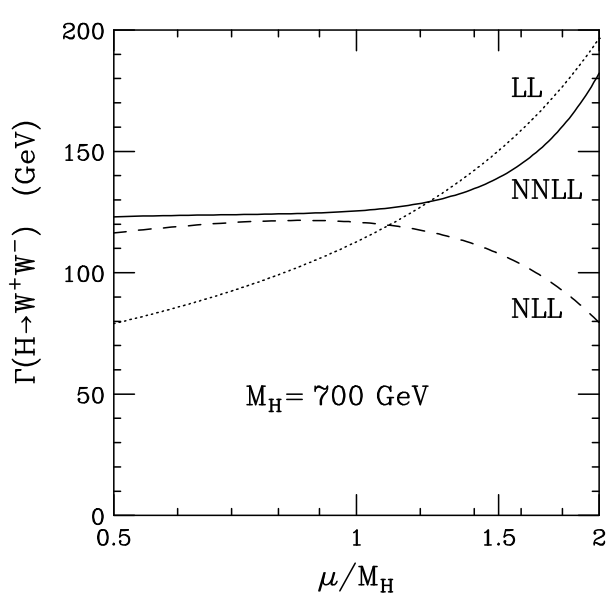

Figure 3. The decay width of $H \rightarrow W_{L}^{+} W_{L}^{-}$ varying the scale $\mu$. The Higgs mass is fixed to be $700 \mathrm{GeV}$.

The $\mu$-dependence of the two-loop width (3) is given in [9]. Fixing the Higgs mass to be $700 \mathrm{GeV}$, the result for the decay channel $H \rightarrow W^{+} W^{-}$is given in Fig. 3. The scale $\mu=M_{H}$ seems to be a good choice. A major modification like in the case of high-energy scattering processes is not necessary.

\section{Summary}

If the Higgs mass is large, perturbative highenergy cross sections such as $\sigma\left(W_{L}^{+} W_{L}^{-} \rightarrow Z_{L} Z_{L}\right)$ are sensitive to the choice of the renormalization scale $\mu$. The usual choice $\mu=\sqrt{s}$ can lead to unreliable perturbative results for relatively small Higgs mass and coupling. Motivated by the bubble structure of the contributing Feynman diagrams, we find the choice $\mu=\sqrt{s} / \mathrm{e} \approx \sqrt{s} / 2.7$ to yield an approximate resummation of specific terms in the perturbative expansion. As a result we obtain reliable perturbative predictions even for Higgs couplings as large as $\lambda(\sqrt{s} / \mathrm{e}) \approx 4$, a significant improvement over the previous bound of $\lambda(\sqrt{s}) \approx 2.2$. In the case of Higgs decays no dominant bubble contributions exist, and the standard choice $\mu=M_{H}$ is the right choice. 


\section{Acknowledgements}

I thank the theory group of DESY-IfH Zeuthen for inviting me to this workshop. It was a pleasure to introduce the recent work on radiative corrections in the SM Higgs sector to the experts of the QED and QCD community. Discussions with many participants of the workshop are gratefully acknowledged, especially conversations with K.G. Chetyrkin and A. Davydychev. Further thanks go to my collaborator Scott Willenbrock.

\section{REFERENCES}

1. W. Beenakker et. al., Cern Yellow Report No. CERN-96-01 and hep-ph/9602351; F. Boudjema et. al., Report No. ROME1-112795 and hep-ph/9601224. M. Carena, P. Zerwas (conveners) et. al., Cern Yellow Report No. CERN-96-01 and hep-ph/9602250.

2. J. Bagger et. al., Phys. Rev. D49, 1246 (1994); ibid 52, 3878 (1995).

3. F. Boudjema and E. Chopin, Report No. ENSLAPP-A-534-95 (July 1995) and hepph/9507396.

4. V.A. Ilin et. al., Report No. KEK-CP-030 (June 1995) and hep-ph/9506326.

5. A. Djouadi et. al., Report No. KA-TP16-96 and hep-ph/9605437; M. Baillargeon et. al., Report No. UB-HET-96-01 and hep$\mathrm{ph} / 9603220$.

6. L. Durand, J.M. Johnson, and J.L. Lopez, Phys. Rev. Lett. 64, 1215 (1990); Phys. Rev. D 45, 3112 (1992).

7. L. Durand, P. Maher, and K. Riesselmann, Phys. Rev. D 48, 1084 (1993).

8. K. Riesselmann, Report No. TUM-HEP223/95 and hep-ph/9507413; to appear in Phys. Rev. D.

9. U. Nierste and K. Riesselmann, TUM Report No. TUM-HEP-224/95 and hep-ph/9511407 (November 1995); to appear in Phys. Rev. D. 10. A. Ghinculov, Nucl. Phys. B455, 21 (1995).

11. A. Frink, B.A. Kniehl, D. Kreimer, and K. Riesselmann, Report No. TUM-HEP247/96 (June 1996) and hep-ph/9606310; to appear in Phys. Rev. D.

12. L. Durand, B.A. Kniehl, and K. Riesselmann, Phys. Rev. Lett. 72, 2534 (1994); 74, 1699(E)
(1995); Phys. Rev. D 51, 5007 (1995).

13. A. Ghinculov, Phys. Lett. B 337, 137 (1994); 346, 426(E) (1995).

14. P.N. Maher, L. Durand, and K. Riesselmann, Phys. Rev. D 48, 1061 (1993); 52, 553(E) (1995).

15. S. Dawson and S. Willenbrock, Phys. Rev. D 40, 2880 (1989).

16. S.N. Gupta, J.M. Johnson, and W.W. Repko, Phys. Rev. D 48, 2083 (1993).

17. M. Veltman and F. Yndurain,, Nucl. Phys. B325, 1 (1989).

18. K. Riesselmann and S. Willenbrock, TU Munich Report No. TUM-HEP-236/96 (August 1996) and hep-ph/9608280.

19. P. Stevenson, Nucl. Phys. B203, 472 (1982).

20. L. Maiani, G. Parisi, and R. Petronzio, Nucl. Phys. B136, 115 (1979); N. Cabbibo, L. Maiani, G. Parisi, and R. Petronzio, Nucl. Phys. B158, 295 (1979); B. Grzadkowski and M. Lindner, Phys. Lett. B178, 81 (1986); M. Lindner, Z. Phys. C31, 295 (1986).

21. M. Lüscher and P. Weisz, Phys. Lett. B212, 472 (1988); Nucl. Phys. B318, 705 (1989); M. Göckeler, H. Kastrup, T. Neuhaus, and F. Zimmermann, Nucl. Phys. B404, 517 (1993); U. Heller, M. Klomfass, H. Neuberger, and P. Vranas, Nucl. Phys. B405, 555 (1993); M. Göckeler, H.A. Kastrup, J. Westphalen, and F. Zimmermann, Nucl. Phys. B425, 413 (1994). 